\title{
Overall survival, costs and healthcare resource use by number of regimens received in elderly patients with newly diagnosed metastatic triple-negative breast cancer
}

\author{
Abdalla Aly ${ }^{1}$, Ruchitbhai Shah ${ }^{* 1}$, Kala Hill ${ }^{2}$ \& Marc F Botteman ${ }^{1}$ \\ ${ }^{1}$ Pharmerit International, 4350 East-West Hwy, Suite 1110, Bethesda, MD 20814, USA \\ ${ }^{2}$ Celldex Therapeutics, 53 I-78 Frontage Rd, Hampton, NJ 08827, USA \\ *Author for correspondence: Tel.: +1 240821 1273; rshah@pharmerit.com
}

\begin{abstract}
Aim: This analysis estimated the overall survival, treatment patterns and economic burden of elderly metastatic triple-negative breast cancer patients. Materials \& methods: Patients ( $\geq 66$ years) with metastatic triple-negative breast cancer were identified from the SEER-Medicare database. Treatment patterns were defined in terms of first, second and third or more regimens. Healthcare resource use and costs were reported over the follow-up period and over the treatment duration of each regimen. Results: A total of $51 \%$ of patients did not receive chemotherapy. Taxanes were most commonly used. Median survival was 7 months. The mean cumulative (per patient per month) cost per patient was US $\$ 73,586$ (US $\$ 10,084$ ). Mean cost in first and second regimen were US $\$ 26,950$ and US $\$ 33,347$. Conclusion: About half of patients did not receive chemotherapy. Receipt of increasing regimens led to higher mean costs and healthcare resource use.
\end{abstract}

First draft submitted: 29 May 2018; Accepted for publication: 19 December 2018; Published online: 5 February 2019

Keywords: chemotherapy $\bullet$ elderly $\bullet$ metastatic disease $\bullet$ SEER-Medicare $\bullet$ triple-negative breast cancer

Triple-negative breast cancer (TNBC) is an aggressive variant of breast cancer that accounts for approximately $15 \%$ of all breast cancer cases [1]. In TNBC, the lack of gene expression for estrogen receptor, progesterone receptor or HER2 greatly limits the treatment options for patients since most conventional chemotherapies target one of the three receptors. The incidence rate of TNBC is highest in non-Hispanic blacks and younger women [2]. However, about one in three TNBC patients are over the age of 65 [3]. Patients with TNBC typically have a worse prognosis in terms of relapse-free survival and overall survival compared with patients of a primarily non-TNBC subtype [4]. While TNBC patients may respond initially to anthracycline/taxane-based adjuvant chemotherapies, they often develop visceral or CNS metastasis [5]. TNBC patients have an increased likelihood of experiencing a distant recurrence compared with other breast cancer patients, which peak at 3-years post-treatment, and the majority of deaths in TNBC occur within 5 years of diagnosis [6]. In addition, TNBC patients have a very poor prognosis from the time of recurrence, with survival rarely extending beyond 12 months $[7,8]$.

Despite the suboptimal outcomes, conventional chemotherapy remains the mainstay in metastatic triple-negative breast cancer (mTNBC) treatment. Nonetheless, traditional chemotherapy for patients who have relapsed are limited since these patients usually have become resistant to anthracyclines or taxanes, which are typically used in the neoadjuvant and adjuvant setting [9]. Response to chemotherapy is highly heterogeneous and depends on patient age and other characteristics. In the metastatic setting, the duration of response to chemotherapy for TNBC patients is usually short (12 weeks after first-line treatment, 9 weeks after second-line and 4 weeks after third-line treatment) [10,11]. Frail older patients may benefit from chemotherapy; however, in most cases, these patients are generally considered for palliative and hospice care [12]. Recent developments in the treatment of mTNBC have focused on targeted therapies including targeting receptors such as the androgen receptor and the epidermal growth factor receptor, inhibiting enzymes/proteins such as the ADP ribose polymerase and the glycoprotein NMB, or

Future Medicine 
inhibiting a molecular pathway such as the PI3K/Akt/mTOR [5]. These targeted therapies are quickly changing the treatment paradigm for mTNBC.

Most literature evaluating the outcomes of TNBC have focused on survival and recurrence of disease [4,13-15]. To date, literature on the overall economic burden and healthcare resource use (HCRU) of mTNBC remains limited. Baser and colleagues examined the survival and healthcare utilization costs for women with TNBC compared with non-TNBC breast cancer using a US managed care registry [16]. Their analysis provided insights on the economic burden of TNBC and observed that patients with TNBC incurred higher healthcare costs, and had more hospital admissions and ER visits compared with their non-TNBC counterparts [16]. Another recent analysis in the elderly found that stage IV TNBC was more resource intensive and costly to Medicare than stage III TNBC [17]. However, given the evolving treatment landscape of $\mathrm{mTNBC}$ with the emergence of targeted therapies, more information is needed on the impact that each sequential regimen has on the costs and HCRU of mTNBC.

Since most mTNBC patients are likely to require several regimens over the course of their disease, it is important to understand the treatment patterns and relative costs and healthcare utilization of each sequential regimen as this will provide a reference for healthcare providers to assess the value and impact that the new innovative therapies may have along the treatment trajectory. This analysis aimed to describe the treatment patterns and overall survival of mTNBC patients according to the number of regimens received and examine the healthcare costs and HCRU of patients with $\mathrm{mTNBC}$ according to the number of regimens received.

\section{Materials \& methods}

An observational retrospective analysis was conducted using SEER-Medicare database [14]. The SEER-Medicare database comprise data from the National Cancer Institute's SEER cancer registries which consolidates data from state and regional registries covering $28 \%$ of the US population [16] and the Medicare enrollment and claims files from the Centers for Medicare and Medicaid Services (CMS) [14]. The linked dataset provides demographic and clinical characteristics at diagnosis and longitudinal information on services used [18].

\section{Inclusion criteria}

Patients newly diagnosed with mTNBC as their primary cancer between 2004 and 2011 were identified. Triplenegative status was obtained from the SEER registry, except for HER2 status, which was unavailable from 2004 to 2009, during which time patients were presumed to be HER2 negative if they had a claim for a HER2 test followed by absence of hormonal therapy, an approach that has been previously used [19,20]. Patients were included in this study if they were $\geq 66$ years-old at the time of diagnosis, and had continuous enrollment in Medicare Parts A and B in the 12 months prior to diagnosis (to allow for ascertainment of baseline clinical characteristics). Patients were excluded if they had an unknown diagnosis date, received a post mortem diagnosis of mTNBC, were diagnosed with another type of cancer in the 5 years prior to diagnosis, died within 30 days of diagnosis (to allow for the opportunity to receive treatment), or were enrolled in a health maintenance organization (HMO) in the 12 months prior to diagnosis. The study sample was followed up from the date of mTNBC diagnosis until death, loss of Medicare enrollment, HMO enrollment, or end of study period (31 December 2013), whichever occurred first. Included patients were categorized into four mutually exclusive groups: no chemotherapy (NC), patients who received only one regimen $(1 \mathrm{R})$, patients who received only two regimens $(2 \mathrm{R})$ and patients who received three or more regimens $(3 \mathrm{R}+)$

\section{Patient characteristics at baseline}

Patients' characteristics were assessed during the baseline study period, in other words, 12 months prior to mTNBC diagnosis. Sociodemographic characteristics included in this study included age at diagnosis, race/ethnicity, census region and marital status. Baseline comorbidities of the study sample were measured based on the Deyo and Romano adaptation of the Charlson Comorbidity Index (CCI) using Medicare claims. A proxy measure for poor performance status was created and defined as any claim in the baseline period for wheelchair use, oxygen use, walking aid, hospital bed, hospice, skilled nursing facility or hospitalization [21-24]. Baseline cancer and clinical characteristics assessed included diagnosis year, topographic location of tumor, tumor size and laterality.

\section{Treatment patterns}

Treatment patterns were characterized using an algorithm used previously in breast cancer and other cancers [25-27]. The first regimen was the first chemotherapy used post diagnosis. A subsequent regimen was defined as: a new 

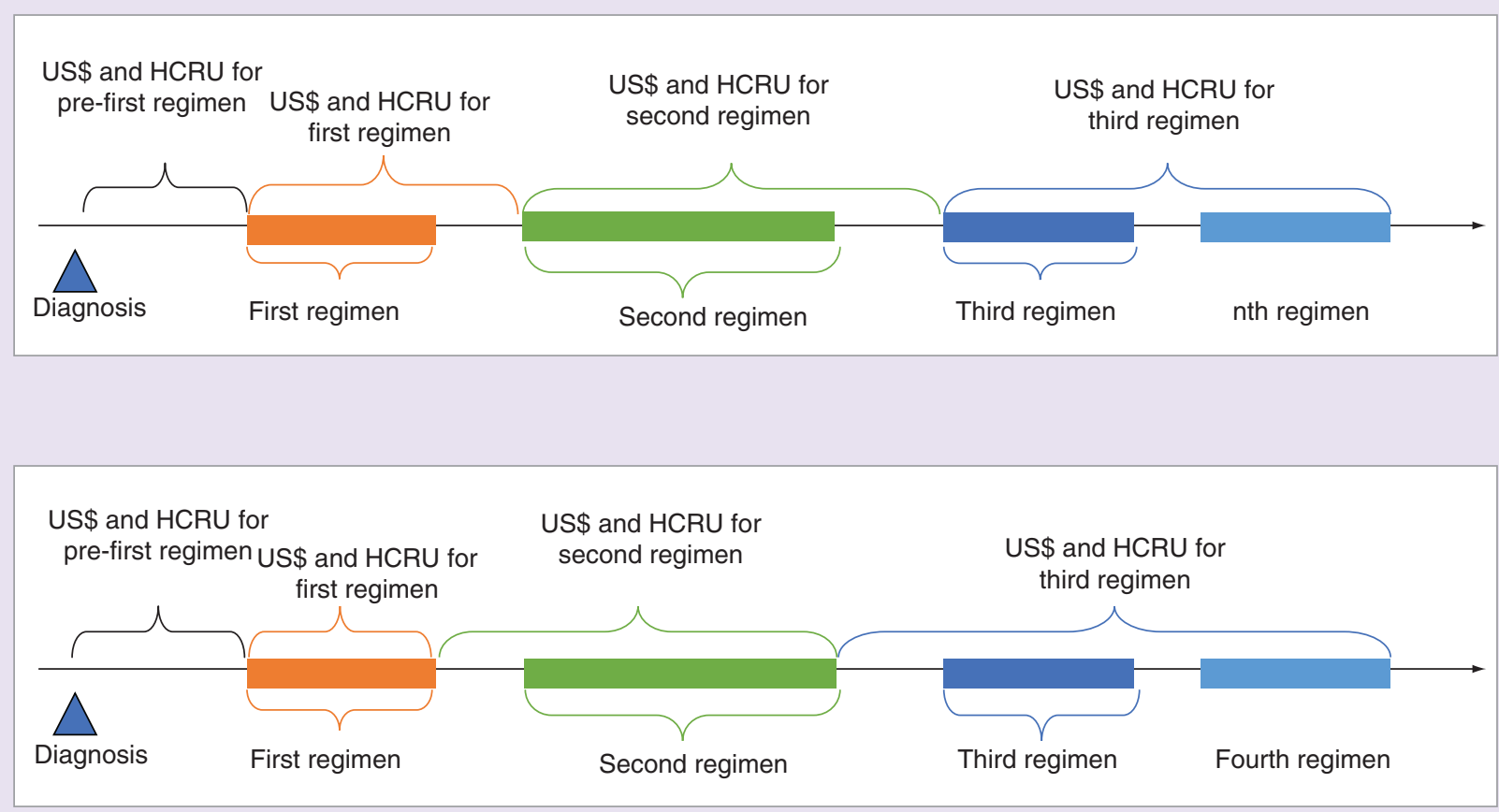

Figure 1. Cost attribution to each line of treatment. According to main (top panel) and sensitivity analysis (bottom panel). HCRU: Healthcare resource use.

chemotherapy added more than 14 days after the start date of the prior chemotherapy, or the same chemotherapy agent was rechallenged after a 90-day gap from the first time it was used. We described treatments patterns according to individual agents as well as mechanism of action following the classification by Zeichner $e t$ al. [28]

\section{Overall survival}

The time from mTNBC diagnosis to mortality was assessed and compared between NC, $1 \mathrm{R}, 2 \mathrm{R}$ and $3 \mathrm{R}+$ patients. The date of diagnosis was available from the SEER data; however, since SEER only provides the month and year of diagnosis, we imputed to the fifteenth of the calendar month and analyses were adjusted to account for the 15-day error.

\section{HCRU \& costs}

Direct medical costs, in terms of Medicare reimbursed amounts within claims, were categorized as inpatient, outpatient, physician, home health, hospice, durable medical equipment (DME) and prescription drug costs. The intent of this analysis was to estimate the economic burden in mTNBC patients and to examine these costs during the course of the treatment pathways. Therefore, costs were reported in two ways. First, cumulative mean costs per patient and per patient per month (PPPM) in the full sample and in the NC, 1R, 2R and 3R+ patients; and second, mean costs per patient in four phases during the follow-up period: from diagnosis to start of treatment (pretreatment phase), from start of first regimen to start of second regimen (first regimen duration), from start of second regimen to start of third regimen (second regimen duration) and from start of third regimen to end of treatment (third and subsequent regimens duration; Figure 1, top panel). Since costs incurred between regimens may arguably be attributed to the previous regimen (e.g., managing/treating toxicities that may have been due to a previous regimen) or the subsequent regimen (e.g., monitoring for progression that requires a subsequent regimen), we conducted a sensitivity analysis (SA) that used a different cost attribution method (Figure 1, bottom panel). All costs were reported in 2017 US dollars.

HCRU items were classified into admissions/visits (emergency room [ER], hospital admissions and length of stay, intensive care unit $[\mathrm{ICU}]$ admissions, outpatient office visits) and supportive care (radiation therapy, occupational therapy, physical therapy and speech therapy). Additionally, the proportion of patients receiving prescription drug medications (antiemetics, anti-anxiety drugs, narcotic opioids, sedatives/hypnotics, steroids) were reported as mean 


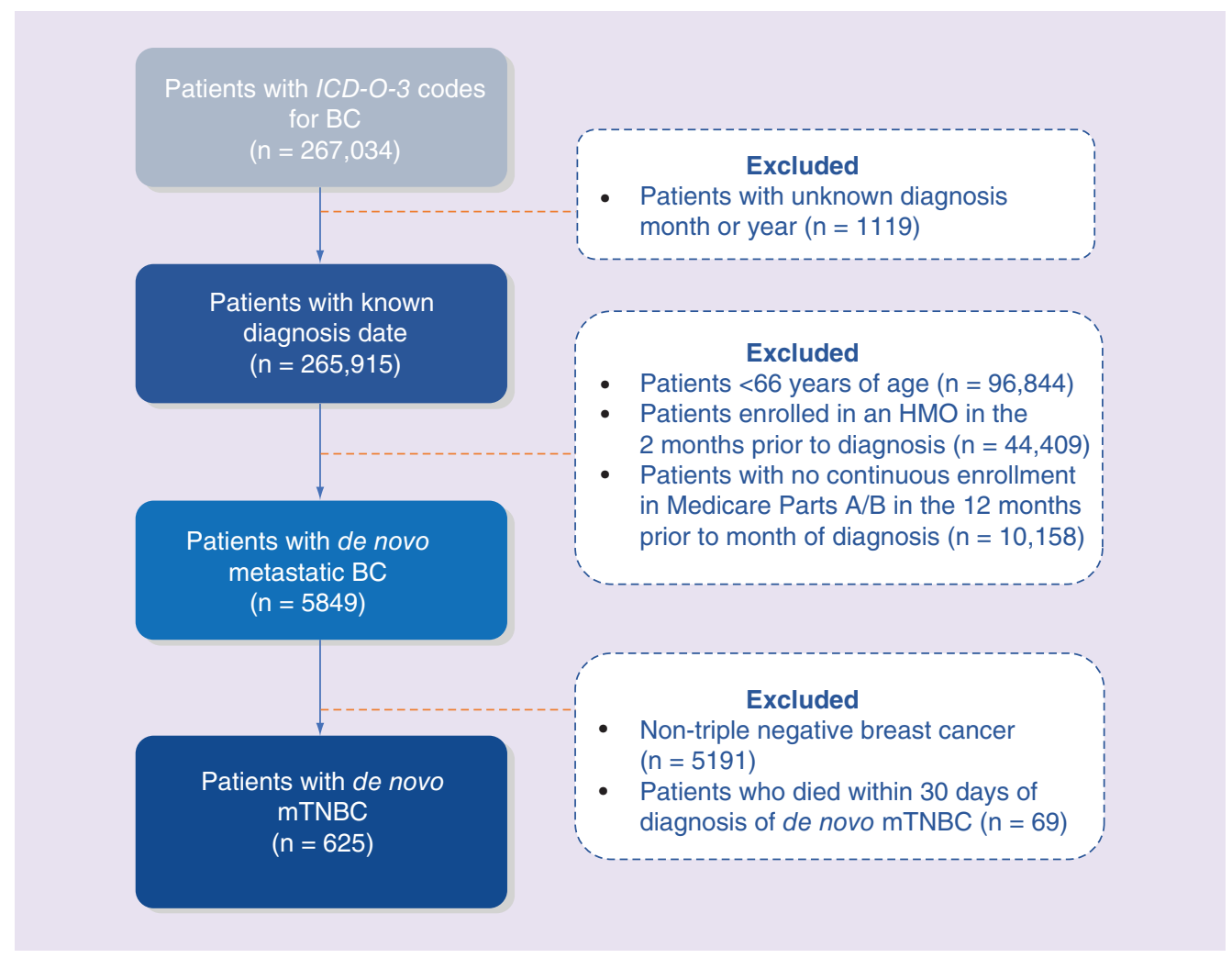

Figure 2. Patient attrition flowchart.

BC: Breast cancer; HMO: Health maintenance organization; ICD-O-3: International Classification of Diseases for Oncology, Version 3; mTNBC: Metastatic triple-negative breast cancer.

(standard deviation [SD]) and median (first quartile [Q1] to third quartile [Q3]) per patient over the four phases as described previously for costs.

\section{Statistical analysis}

Bivariate analyses were used to compare baseline characteristics, costs and HCRU between NC, 1R, 2R and 3R+ patients. For categorical variables, frequency and percentage distributions were reported. Statistical comparisons were conducted between cohorts using Pearson $\chi^{2}$ tests or Fisher's exact tests when any frequencies were $<5$. For continuous variables, median, interquartile range (IQR), mean and SD were reported. The Kolmogorov-Smirnov test was used to test the distribution of the variables. T-tests were used for normally distributed variables and Mann-Whitney tests for not normally distributed variables. Treatment patterns were described in terms of the chemotherapy used in the first, second and third regimen, median time from mTNBC diagnosis to start of regimen and the median duration within and between each regimen. Multinomial logistic regression was used to assess predictors of the number of regimens received and presented in terms of odds ratios (ORs) and 95\% CIs. The Kaplan-Meier method was used to examine the time from diagnosis to death comparing between NC, 1R, 2R and $3 \mathrm{R}+$ patients. The 95\% CI was reported using Brookmeyer and Crowley methodology [29]. SAS 9.4 was used for all analyses.

\section{Results}

\section{Study sample}

Of 625 patients diagnosed with mTNBC who met the inclusion criteria (Figure 2), the median age at diagnosis was 76 years (range: 66-102 years); and the majority were non-Hispanic white (72\%), lived in an urban location (88\%) and had a CCI score greater than $1(25 \%)$, and all were women. Patients were followed up for 14.1 months on average. Fifty-one percent of the study sample received chemotherapy treatment during study follow-up. Compared with chemotherapy-treated patients, the untreated patients were older, had higher CCI and had poor performance 
Overall survival, costs \& HCRU by number of regimens received in elderly patients with newly diagnosed mTNBC Research Article

Table 1. Baseline sociodemographic and clinical characteristics of study sample. ${ }^{\dagger}$

\begin{tabular}{|c|c|c|c|c|c|c|}
\hline Baseline characteristics & All patients $(n=625)$ & $\begin{array}{l}\text { No chemotherapy } \\
(n=308)\end{array}$ & $1 R$ only $(n=161)$ & $2 R$ only $(n=88)$ & $3 R+(n=68)$ & p-value \\
\hline Age at diagnosis, mean (SD), years & $76.8(7.3)$ & $79.0(7.7)$ & $75.7(6.6)$ & $73.5(5.0)$ & $73.7(5.8)$ & $<0.001$ \\
\hline Race/ethnicity, n (\%): & & & & & & 0.101 \\
\hline - Non-Hispanic white & $454(72 \%)$ & $221(71 \%)$ & $115(71 \%)$ & $69(78 \%)$ & $49(72 \%)$ & \\
\hline - Non-Hispanic black & $119(19 \%)$ & $65(21 \%)$ & $30(18 \%)$ & $11(12 \%)$ & $13(19 \%)$ & \\
\hline - Hispanic & $38(6 \%)$ & $11(3 \%)$ & NR & NR & NR & \\
\hline - Other & $14(2 \%)$ & $11(3 \%)$ & NR & NR & NR & \\
\hline Marital status at diagnosis, n (\%): & & & & & & $<0.001$ \\
\hline - Married & $184(29 \%)$ & $67(21 \%)$ & $48(29 \%)$ & $35(39 \%)$ & $34(50 \%)$ & \\
\hline - Separated/divorced/widowed & $351(56 \%)$ & $188(61 \%)$ & $91(56 \%)$ & $42(47 \%)$ & $30(44 \%)$ & \\
\hline - Single (never married) & $64(10 \%)$ & $35(11 \%)$ & NR & NR & NR & \\
\hline - Unknown & $26(4 \%)$ & $18(5 \%)$ & NR & NR & NR & \\
\hline US census location, $\mathrm{n}(\%)$ : & & & & & & 0.195 \\
\hline - Midwest & $98(15 \%)$ & $56(18 \%)$ & $18(11 \%)$ & $12(13 \%)$ & NR & \\
\hline - Northeast & $139(22 \%)$ & $75(24 \%)$ & $37(22 \%)$ & $18(20 \%)$ & NR & \\
\hline - South & $171(27 \%)$ & $75(24 \%)$ & $49(30 \%)$ & $30(34 \%)$ & $17(25 \%)$ & \\
\hline - West & $217(34 \%)$ & $102(33 \%)$ & $57(35 \%)$ & $28(31 \%)$ & $30(44 \%)$ & \\
\hline Location type, n (\%): & & & & & & 0.305 \\
\hline - Rural & $70(11 \%)$ & $28(9 \%)$ & $24(14 \%)$ & NR & NR & \\
\hline - Urban & $555(88 \%)$ & $280(90 \%)$ & $137(85 \%)$ & NR & NR & \\
\hline Charlson Comorbidity Index, n (\%): & & & & & & 0.003 \\
\hline-0 & $331(53 \%)$ & $141(46 \%)$ & $95(59 \%)$ & $51(58 \%)$ & $44(65 \%)$ & \\
\hline-1 & $140(22 \%)$ & $70(23 \%)$ & $29(18 \%)$ & $23(26 \%)$ & $18(26 \%)$ & \\
\hline-2 & $73(12 \%)$ & $46(15 \%)$ & $19(12 \%)$ & NR & NR & \\
\hline$-3+$ & $81(13 \%)$ & $51(17 \%)$ & $18(11 \%)$ & NR & NR & \\
\hline Poor performance status, $\mathrm{n}(\%)$ : & & & & & & 0.016 \\
\hline- No & $495(79 \%)$ & $231(75 \%)$ & $133(83 \%)$ & $69(78 \%)$ & NR & \\
\hline- Yes & $130(21 \%)$ & $77(25 \%)$ & $28(17 \%)$ & $19(22 \%)$ & NR & \\
\hline Tumor laterality, n (\%): & & & & & & 0.311 \\
\hline - Left side & $306(49 \%)$ & $157(51 \%)$ & NR & $43(49 \%)$ & $34(50 \%)$ & \\
\hline - Right side & $286(46 \%)$ & $132(43 \%)$ & $83(52 \%)$ & NR & NR & \\
\hline - Unknown or midline & $33(5 \%)$ & $19(6 \%)$ & NR & NR & NR & \\
\hline Tumor size, mean (SD), mm & $115.5(243)$ & $93.8(203)$ & $131.5(268)$ & $118.5(257)$ & $170.5(314)$ & 0.140 \\
\hline Diagnosis year: & & & & & & 0.112 \\
\hline-2004 & $67(11 \%)$ & $45(15 \%)$ & NR & NR & NR & \\
\hline-2005 & $81(13 \%)$ & $38(12 \%)$ & $18(11 \%)$ & $15(17 \%)$ & NR & \\
\hline-2006 & $94(15 \%)$ & $55(18 \%)$ & $23(14 \%)$ & NR & NR & \\
\hline-2007 & $72(12 \%)$ & $35(11 \%)$ & $21(13 \%)$ & NR & NR & \\
\hline-2008 & $78(12 \%)$ & $35(11 \%)$ & $23(14 \%)$ & NR & NR & \\
\hline-2009 & $96(15 \%)$ & $48(16 \%)$ & $26(16 \%)$ & NR & $12(18 \%)$ & \\
\hline-2010 & $67(11 \%)$ & $29(9 \%)$ & NR & $12(13 \%)$ & NR & \\
\hline-2011 & $70(11 \%)$ & $23(7 \%)$ & $25(16 \%)$ & $12(14 \%)$ & NR & \\
\hline
\end{tabular}

indicators at diagnosis (all $\mathrm{p}<0.05$; Table 1 ). The proportion of patients having congestive heart failure at diagnosis was higher in NC patients (19\%) compared with chemotherapy-treated patients (7\%). 


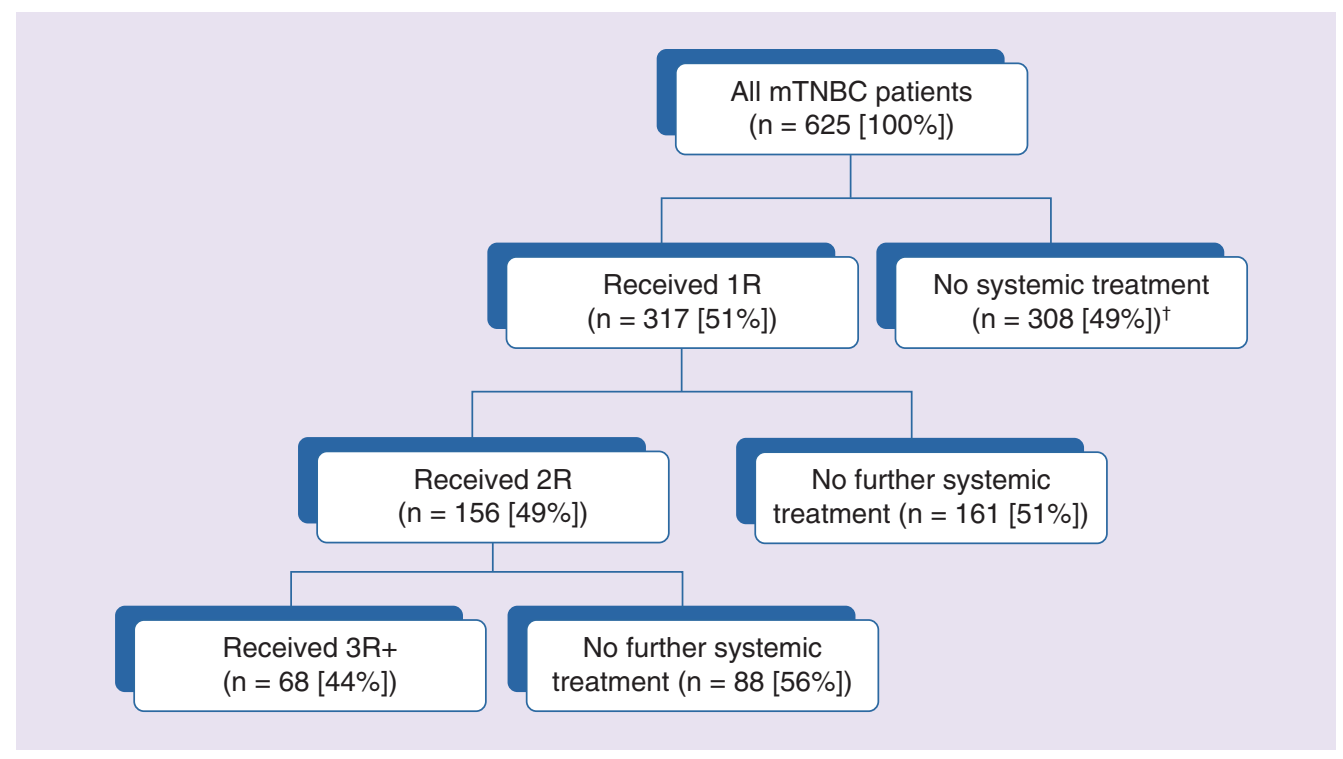

Figure 3. Patient disposition by regimen sequence.

$\dagger 72 / 308(23 \%)$ got radiation in the untreated group.

1R: Only one regimen; 2R: Only two regimens; 3R+: Three or more regimens; mTNBC: Metastatic triple-negative breast cancer.

\begin{tabular}{|c|c|c|c|}
\hline Treatments received & First regimen $(n=317)$ & Second regimen $(n=156)$ & Third regimen $(n=68)$ \\
\hline \multicolumn{4}{|c|}{ Single versus combination, $\mathrm{n}(\%)$ : } \\
\hline - Single agent & $205(65 \%)$ & $74(47 \%)$ & $40(59 \%)$ \\
\hline - Combination regimens & $112(35 \%)$ & $82(53 \%)$ & $28(41 \%)$ \\
\hline \multicolumn{4}{|c|}{ Mechanism of action, $\mathrm{n}$ (\% of single agents): } \\
\hline - Taxane & $80(39 \%)$ & $23(31 \%)$ & $\mathrm{NR}^{\dagger}$ \\
\hline - Anthracyclines & $29(14 \%)$ & $13(18 \%)$ & $\mathrm{NR}^{\dagger}$ \\
\hline - Antimetabolites/others & $96(47 \%)$ & $38(51 \%)$ & $27(73 \%)$ \\
\hline \multicolumn{4}{|c|}{ Individual agents, $\mathrm{n}$ (\% of single agents): } \\
\hline - Paclitaxel & $72(35 \%)$ & $18(24 \%)$ & $\mathrm{NR}^{\dagger}$ \\
\hline - Capecitabine & $51(25 \%)$ & $20(27 \%)$ & $\mathrm{NR}^{\dagger}$ \\
\hline - Doxorubicin & $27(13 \%)$ & $13(18 \%)$ & $\mathrm{NR}^{\dagger}$ \\
\hline - Gemcitabine & $\mathrm{NR}^{\dagger}$ & $\mathrm{NR}^{\dagger}$ & $13(35 \%)$ \\
\hline
\end{tabular}

\section{Treatment patterns}

Of the 625 patients included in the study sample, $49 \%$ received chemotherapy. Of the patients receiving chemotherapy, $51 \%$ of the mTNBC patients received $1 \mathrm{R}$ only, $28 \%$ received $2 \mathrm{R}$ only and $21 \%$ received $3 \mathrm{R}+$ (Figure 3 ). The majority of the first regimens used by mTNBC patients were single chemotherapeutic agents (65\%). Of the single agents used, antimetabolites/others (47\%) were the most common, followed by taxanes (39\%) and anthracyclines (14\%). The proportions of patients using combination chemotherapy were higher in the second (53\%) and third (41\%) regimens compared with the first regimen (35\%; Table 2). The median durations of the first, second and third regimens were 2.7, 3.1 and 2.3 months, respectively. Patients started their first regimen approximately after a median of 1.6 months following diagnosis; the median times to start of the second and third regimens after the end of the previous regimen were 4.6 and 6.2 months, respectively.

Results from the multinomial logistic regression model suggested that age, marital status, comorbidity burden and tumor size were strong predictors of receipt of more regimens (Table 3). Specifically, compared with patients 


\begin{tabular}{|c|c|c|c|c|}
\hline \multirow{2}{*}{ Factor } & \multirow{2}{*}{ Category } & \multicolumn{3}{|c|}{ Odds ratio $(95 \% \mathrm{Cl})$} \\
\hline & & $1 R$ only $(n=317)$ & $2 R$ only $(n=156)$ & $3 R+(n=68)$ \\
\hline \multirow[t]{4}{*}{ Age at diagnosis, years } & $66-70$ & Reference & & \\
\hline & $71-75$ & $0.86(0.47-1.57)$ & $0.76(0.39-1.5)$ & $0.71(0.33-1.51)$ \\
\hline & $76-80$ & $0.77(0.42-1.38)$ & $0.57(0.28-1.14)$ & $0.34(0.14-0.79)$ \\
\hline & $80+$ & $0.37(0.2-0.66)$ & $0.13(0.06-0.31)$ & $0.21(0.09-0.48)$ \\
\hline \multirow{3}{*}{ Marital status at diagnosis } & Married & $1.34(0.58-1.29)$ & $2.72(1.05-7.04)$ & $6.27(1.72-22.86)$ \\
\hline & Separated/divorced/widowed & $1.12(0.58-2.16)$ & $1.68(0.67-4.23)$ & $0.13(0.06-0.31)$ \\
\hline & Unknown & $0.32(0.08-1.29)$ & $1.58(0.38-6.68)$ & $0.62(0.06-6.84)$ \\
\hline \multirow[t]{2}{*}{ US census location } & West & Reference & & \\
\hline & South & $1.29(0.77-2.15)$ & $1.65(0.87-3.11)$ & $0.97(0.47-1.99)$ \\
\hline \multirow{2}{*}{ Charlson Comorbidity Index } & 2 & $0.65(0.34-1.26)$ & $0.25(0.09-0.73)$ & $0.28(0.08-1.03)$ \\
\hline & $3+$ & $0.69(0.34-1.39)$ & $0.51(0.2-1.31)$ & $0.38(0.1-1.46)$ \\
\hline \multirow[t]{2}{*}{ Poor performance status } & No & Reference & & \\
\hline & Yes & $0.78(0.44-1.37)$ & $1.15(0.57-2.32)$ & $0.43(0.16-1.17)$ \\
\hline \multirow[t]{4}{*}{ Tumor size (categorical) } & $<50 \mathrm{~mm}$ & Reference & & \\
\hline & $50-70 \mathrm{~mm}$ & $0.97(0.56-1.68)$ & $0.98(0.52-1.86)$ & $1.27(0.57-2.86)$ \\
\hline & $>70 \mathrm{~mm}$ & $1.45(0.79-2.64)$ & $1.22(0.58-2.59)$ & $2.66(1.14-6.19)$ \\
\hline & Missing & $1.32(0.71-2.44)$ & $0.52(0.22-1.26)$ & $1.53(0.6-3.9)$ \\
\hline
\end{tabular}

1R: Patients who received only one regimen; $2 \mathrm{R}$ : Patients who received only two regimens; $3 R+$ : Patients who received three or more regimens.

who were 66-70 years-old at diagnosis, patients > 80 years of age at diagnosis were 63\% (OR: 0.37; 95\% CI: 0.2-0.66) less likely to receive $1 \mathrm{R}$ only (OR: 0.13 ; 95\% CI: $0.06-0.31$ ), $87 \%$ less likely to receive $2 \mathrm{R}$ only (OR: 0.21; 95\% CI: 0.09-0.48) and 79\% less likely to receive 3R+ (OR: 0.21; 95\% CI: 0.09-0.48). Similarly, patients who were 76-80 years-old at diagnosis were 66\% (OR: 0.34; 95\% CI: 0.14-0.79) less likely to receive 3R+ versus patients who were 66-70 years-old at diagnosis (OR: 0.34; 95\% CI: 0.14-0.79). Compared with those who were single (i.e., never married), those who were married were almost three-times as likely to receive $2 \mathrm{R}$ only (OR: 2.72; 95\% CI: 1.05-7.04), and more than six-times as likely to receive 3R+ (OR: 6.27; 95\% CI: 1.72-22.86). Compared with patients having a CCI score of 0 at baseline, those with a CCI of 2 were $75 \%$ (OR: 0.25 ; $95 \%$ CI: $0.09-0.73)$ less likely to receive $2 \mathrm{R}$ only. Finally, compared with patients with a tumor diameter $<50 \mathrm{~mm}$, those with a tumor diameter greater than $70 \mathrm{~mm}$ were about 2.5 -times more likely to receive 3R+ (OR: 2.66; 95\% CI: $1.14-6.19)$.

Overall survival

The median (95\% CI) overall survival for all patients in the study sample was 7 (6.2-8.1) months. Median (95\% CI) survival for all treated patients was 12.2 (10.7-14.9) months compared to 3.5 (3.2-4.3) months for untreated patients. 1R, 2R and 3R+ patients had median (95\% CI) survival of 7.1 (6.3-9.1), 16.0 (13.6- 19.0) and 25.3 (21.6-29.1) months, respectively (Figure 4).

\section{Healthcare costs}

During entire follow-up

The mean cumulative cost per patient was US\$73,586 for all patients and increased as patients received more regimens (NC: US\$51,070; 1R: US $\$ 68,899 ; 2$ R: US $\$ 107,214 ; 3 R+$ : US $\$ 143,150)$. However, the mean PPPM cost was US $\$ 10,084$ for all patients and decreased as the number of regimens received increased (NC: US $\$ 12,101$; 1R: US\$9721; 2R: US\$6853; 3R+: US\$5986; Table 4). Inpatient and physician office visits were the biggest drivers of PPPM costs across all groups. However, the contribution of inpatient and physician costs to the total 


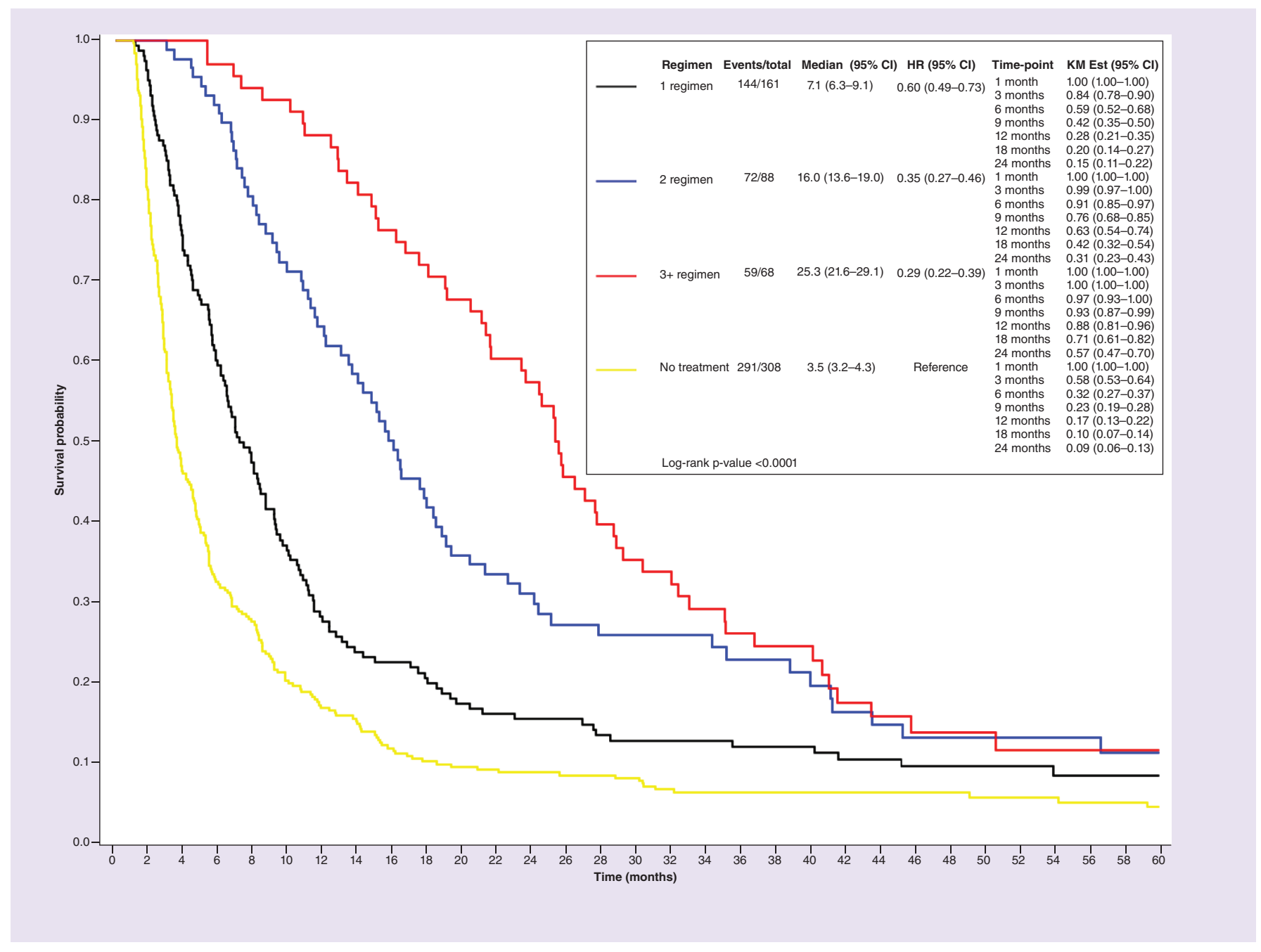

Figure 4. Kaplan-Meier survival curves of study sample stratified by the number of regimens received over the follow-up period. Est: Estimate; HR: Hazard ratio; KM: Kaplan-Meier.

costs depended on the number of regimens received. For example, inpatient costs dropped from $54 \%$ in the NC group to $26 \%$ in the $3 \mathrm{R}+$ group and physician costs increased from $18 \%$ in the NC group to $42 \%$ in the $3 \mathrm{R}+$ group. Similarly, outpatient costs contributed more to the total costs as patients received additional regimens, increasing from $7 \%$ in the NC group to $21 \%$ in the $3 \mathrm{R}+$ group. Across the chemotherapy-treated groups, home health, hospice, durable medical equipment and prescription drug costs accounted for $<10 \%$ of the total costs. However, in the NC group, hospice costs were the highest (11\%) compared with any of the treated groups (2-4\%). The end-of-life costs among chemotherapy-treated patients were US $\$ 5966$ (last 30 days) and US $\$ 14,311$ (last 3 months); $70 \%$ of these costs were in the inpatient setting.

\section{By phase of therapy}

Total unadjusted costs incurred per patient during each treatment phase were US $\$ 13,188$ for pretreatment, US $\$ 26,950$ for the first regimen, US $\$ 33,347$ for the second regimen and US $\$ 50,627$ for third and subsequent regimens. The costs were primarily driven by physician claims (35\% in pre-treatment, $42 \%$ for first regimen, $37 \%$ for second regimen and $48 \%$ for third and subsequent regimens). In sensitivity analyses, the costs per phase were US $\$ 22,423$ for the first regimen, US $\$ 36,549$ for the second regimen and US $\$ 64,453$ for third and subsequent regimens. 


\begin{tabular}{|c|c|c|c|c|c|c|}
\hline Cost & $\begin{array}{l}\text { All patients, } \mathrm{n}=625 \\
\text { Mean (SD) }\end{array}$ & $\begin{array}{l}\text { No chemo., } \mathrm{n}=308 \\
\text { Mean (SD) }\end{array}$ & $\begin{array}{l}1 \mathrm{R} \text { only, } \mathrm{n}=161 \\
\text { Mean (SD) }\end{array}$ & $\begin{array}{l}2 \mathrm{R} \text { only, } \mathrm{n}=88 \\
\text { Mean (SD) }\end{array}$ & $\begin{array}{l}3 R+, n=68 \\
\text { Mean (SD) }\end{array}$ & $\mathrm{p}$-value \\
\hline Total PPPM & $\begin{array}{l}\text { US } \$ 10,084 \\
\text { (US } \$ 10,377 \text { ) }\end{array}$ & $\begin{array}{l}\text { US } \$ 12,101 \\
\text { (US } \$ 12,215)\end{array}$ & US $\$ 9721$ (US\$9979) & US $\$ 6853$ (US $\$ 3919)$ & US\$5986 (US\$3424) & $<0.001$ \\
\hline Inpatient PPPM & US $\$ 5466$ (US $\$ 8403)$ & US $\$ 7414$ (US $\$ 9918)$ & US\$4998 (US\$7906) & US $\$ 2345$ (US $\$ 2600)$ & US\$1789 (US\$2085) & $<0.001$ \\
\hline Outpatient & $\begin{array}{l}\text { US } \$ 11,307 \\
\text { (US } \$ 20,522 \text { ) }\end{array}$ & US $\$ 3767$ (US $\$ 6959)$ & $\begin{array}{l}\text { US } \$ 13,120 \\
\text { (US } \$ 17,918)\end{array}$ & $\begin{array}{l}\text { US } \$ 19,590 \\
\text { (US } \$ 28,077)\end{array}$ & $\begin{array}{l}\text { US } \$ 30,450 \\
\text { (US } \$ 34,333)\end{array}$ & $<0.001$ \\
\hline Outpatient PPPM & US\$1009 (US\$1584) & US $\$ 748$ (US\$1743) & US $\$ 1382$ (US\$1458) & US $\$ 1084$ (US $\$ 1300)$ & US\$1209 (US\$1226) & $<0.001$ \\
\hline Physician & $\begin{array}{l}\text { US } \$ 21,353 \\
\text { (US } \$ 29,372 \text { ) }\end{array}$ & US $\$ 9270$ (US $\$ 8934$ ) & $\begin{array}{l}\text { US } \$ 17,754 \\
\text { (US } \$ 16,333)\end{array}$ & $\begin{array}{l}\text { US } \$ 40,587 \\
\text { (US } \$ 32,777 \text { ) }\end{array}$ & $\begin{array}{l}\text { US } \$ 59,716 \\
\text { (US } \$ 54,358)\end{array}$ & $<0.001$ \\
\hline Home Health PPPM & US $\$ 360$ (US $\$ 789$ ) & US $\$ 440$ (US $\$ 1009$ ) & US $\$ 304$ (US $\$ 525)$ & US $\$ 324$ (US $\$ 519)$ & US $\$ 180$ (US $\$ 251)$ & 0.476 \\
\hline Hospice & US $\$ 4437$ (US $\$ 9938)$ & US $\$ 5504$ (US\$12,174) & US $\$ 3067$ (US $\$ 6746)$ & US $\$ 4094$ (US $\$ 8167$ ) & US $\$ 3292$ (US $\$ 5850)$ & 0.121 \\
\hline Hospice PPPM & US $\$ 629$ (US $\$ 1156)$ & US $\$ 969$ (US\$1467) & US $\$ 364$ (US\$664) & US $\$ 308$ (US $\$ 555)$ & US $\$ 130$ (US $\$ 209$ ) & $<0.001$ \\
\hline Part $D$ drug cost & US $\$ 2289$ (US $\$ 11,507)$ & US $\$ 2050$ (US $\$ 14,889)$ & US $\$ 1454$ (US\$3536) & US $\$ 3725$ (US $\$ 8842$ ) & US $\$ 3487$ (US $\$ 8943$ ) & $<0.001$ \\
\hline Part D drug cost PPPM & US $\$ 165$ (US $\$ 423)$ & US $\$ 158$ (US $\$ 376)$ & US $\$ 190$ (US $\$ 552)$ & US $\$ 169$ (US $\$ 328)$ & US $\$ 135$ (US $\$ 390)$ & 0.063 \\
\hline DME & US $\$ 1891$ (US $\$ 5514)$ & US $\$ 677$ (US $\$ 2346$ ) & US $\$ 2257$ (US $\$ 7925)$ & US $\$ 3191$ (US $\$ 6721$ ) & US $\$ 4841$ (US $\$ 5578$ ) & $<0.001$ \\
\hline DME PPPM & US $\$ 141$ (US $\$ 316)$ & US $\$ 112$ (US $\$ 338)$ & US $\$ 167$ (US $\$ 303$ ) & US $\$ 170$ (US $\$ 340)$ & US $\$ 173$ (US $\$ 178$ ) & $<0.001$ \\
\hline
\end{tabular}

$\dagger$ All costs presented in 2017 US dollars

1R: Patients who received only one regimen; 2R: Patients who received only two regimens; 3R+: Patients who received three or more regimens; chemo.: Chemotherapy; DME: Durable medical equipment; PPPM: Per patient per month.

\section{Healthcare resource use}

Patients in the NC group had a median (IQR) of one (1-2) ER admission, one (1-2) hospitalization, 0 (0-1) ICU admissions, and eight (4-16) office visits in their follow-up period, as well as a median (IQR) hospital length of stay of 6 (4-11) days. In terms of supportive care, in descending order, many NC patients had physical (64.9\%), occupational (54.2\%), speech $(26.0 \%)$ and radiation therapy $(23.4 \%)$. Of the prescription drugs investigated, the most commonly used medication for the NC patients was narcotic opioids (28.9\%).

Among the chemotherapy-treated patients, patients had a median (IQR) of five (3-8) office visits in the pretreatment phase. Hospitalizations were more prevalent in the third and subsequent regimens, with $66.2 \%$ of patients having a hospitalization in that phase. Median hospital length of stay was $\sim 6$ days in all three phases of treatment. Additionally, $57.4 \%$ of patients had an ER admission and $29.4 \%$ had an ICU admission in the $3 \mathrm{R}+$ phase, the highest of all phases in chemotherapy-treated patients. Antiemetics (89.7\%) and systemic steroids $(77.9 \%)$ were more predominantly used in the $3 \mathrm{R}+$ phase compared with other phases (Table 5 ). Figure 5 shows the mean cost per patient by service type during each regimen.

\section{Discussion}

This analysis examined the treatment patterns, overall survival, healthcare costs and HCRU among mTNBC patients in a real-world setting. This analysis makes a unique contribution to the literature by providing deeper insights into the economic impact of each successive regimen for mTNBC patients. First, about half of women with newly diagnosed mTNBC did not receive any chemotherapy. Second, patients who did not receive chemotherapy were older, had more comorbidities and had a median overall survival of 3.5 months (compared with 12.2 months in those who received chemotherapy). Third, the most commonly used first regimen was paclitaxel followed by capecitabine. Fourth, while patients who continued to receive three or more regimens had expectedly higher cumulative mean costs (driven by inpatient and physician costs), they had lower average monthly costs compared with patients receiving one or two regimens only. Fifth, the chemotherapy-treated group cost Medicare approximately US\$13,000 prior 


\section{Table 5. Healthcare resource use per patient over treatment duration by regimen sequence.}

\begin{tabular}{|c|c|c|c|c|c|}
\hline HCRU & $\begin{array}{l}\text { No chemotherapy } \\
(n=308)\end{array}$ & Pre-treatment $(n=317)$ & First regimen $(n=317)$ & Second regimen $(n=156)$ & $3+$ regimens $(n=68)$ \\
\hline$-\mathrm{N}(\%)$ of patients & $236(76.6 \%)$ & $95(30.0 \%)$ & $130(41.0 \%)$ & 75 (48.1\%) & $39(57.4 \%)$ \\
\hline - Median (IQR) & $1(1-2)$ & $0(0-1)$ & $0(0-1)$ & $0(0-1)$ & $1(0-2)$ \\
\hline \multicolumn{6}{|l|}{ Hospitalizations: } \\
\hline - Median (IQR) & $1(1-2)$ & $0(0-1)$ & $0(0-1)$ & $0(0-1)$ & $1(0-2)$ \\
\hline \multicolumn{6}{|c|}{$\begin{array}{l}\text { Hospital length of stay, } \\
\text { days: }\end{array}$} \\
\hline - Mean (SD) & $7.71(6.0)$ & $6.08(6.2)$ & $7.68(5.1)$ & $8.30(5.9)$ & $6.71(5.1)$ \\
\hline - Median (IQR) & $6(4-9.5)$ & $4(3-6)$ & $6(4-11)$ & $6.5(4-11)$ & $6(2-8)$ \\
\hline - Median (IQR) & $0(0-1)$ & $0(0-0)$ & $0(0-0)$ & $0(0-0)$ & $0(0-1)$ \\
\hline \multicolumn{6}{|l|}{ Office visits: } \\
\hline$-\mathrm{N}(\%)$ of patients & $301(97.7 \%)$ & $295(93.1 \%)$ & $315(99.4 \%)$ & $156(100 \%)$ & $68(100 \%)$ \\
\hline - Mean (SD) & $15.13(21.39)$ & $7.10(8.96)$ & $11.35(11.16)$ & $18.71(21.09)$ & $35.03(33.84)$ \\
\hline - Median (IQR) & $8(4-16)$ & $5(3-8)$ & $8(4-14)$ & $13(7-25)$ & $21(9-58.5)$ \\
\hline \multicolumn{6}{|c|}{ Supportive care sessions } \\
\hline \multicolumn{6}{|l|}{ Radiation therapy: } \\
\hline$-\mathrm{N}(\%)$ of patients & $72(23.4 \%)$ & $47(14.8 \%)$ & $38(12.0 \%)$ & $38(24.4 \%)$ & $27(39.7 \%)$ \\
\hline - Mean (SD) & $0.99(2.35)$ & $0.57(1.69)$ & $0.44(1.47)$ & $1.26(2.74)$ & $2.26(3.43)$ \\
\hline - Median (IQR) & $0(0-0)$ & $0(0-0)$ & $0(0-0)$ & $0(0-0)$ & $0(0-5)$ \\
\hline - Median (IQR) & $0(0-1)$ & $0(0-0)$ & $0(0-0)$ & $0(0-0)$ & $0(0-0)$ \\
\hline \multicolumn{6}{|l|}{ Physical therapy: } \\
\hline$-\mathrm{N}(\%)$ of patients & $200(64.9 \%)$ & $64(20.2 \%)$ & $82(25.9 \%)$ & $53(34.0 \%)$ & $36(52.9 \%)$ \\
\hline - Mean (SD) & $1.90(2.72)$ & $0.38(0.97)$ & $0.47(1.17)$ & $0.74(1.39)$ & $1.32(2.07)$ \\
\hline - Median (IQR) & $1(0-2)$ & $0(0-0)$ & $0(0-1)$ & $0(0-1)$ & $1(0-2)$ \\
\hline \multicolumn{6}{|c|}{$\begin{array}{l}\text { Prescription drug fills, n } \\
\text { (\%) }\end{array}$} \\
\hline Antiemetics & $69(22.4 \%)$ & $98(30.9 \%)$ & $234(73.8 \%)$ & $124(79.5 \%)$ & $61(89.7 \%)$ \\
\hline Anti-anxiety drugs & $33(10.7 \%)$ & $80(25.2 \%)$ & $37(11.7 \%)$ & $29(18.6 \%)$ & $15(22.1 \%)$ \\
\hline Narcotic opioids & $89(28.9 \%)$ & $97(30.6 \%)$ & $45(14.2 \%)$ & $37(23.7 \%)$ & $23(33.8 \%)$ \\
\hline Sedatives/hypnotics & $37(12.0 \%)$ & $80(25.2 \%)$ & $42(13.3 \%)$ & $33(21.2 \%)$ & $18(26.5 \%)$ \\
\hline Steroids & $38(12.3 \%)$ & $38(12.0 \%)$ & $206(65.0 \%)$ & $106(68.0 \%)$ & $53(77.9 \%)$ \\
\hline
\end{tabular}




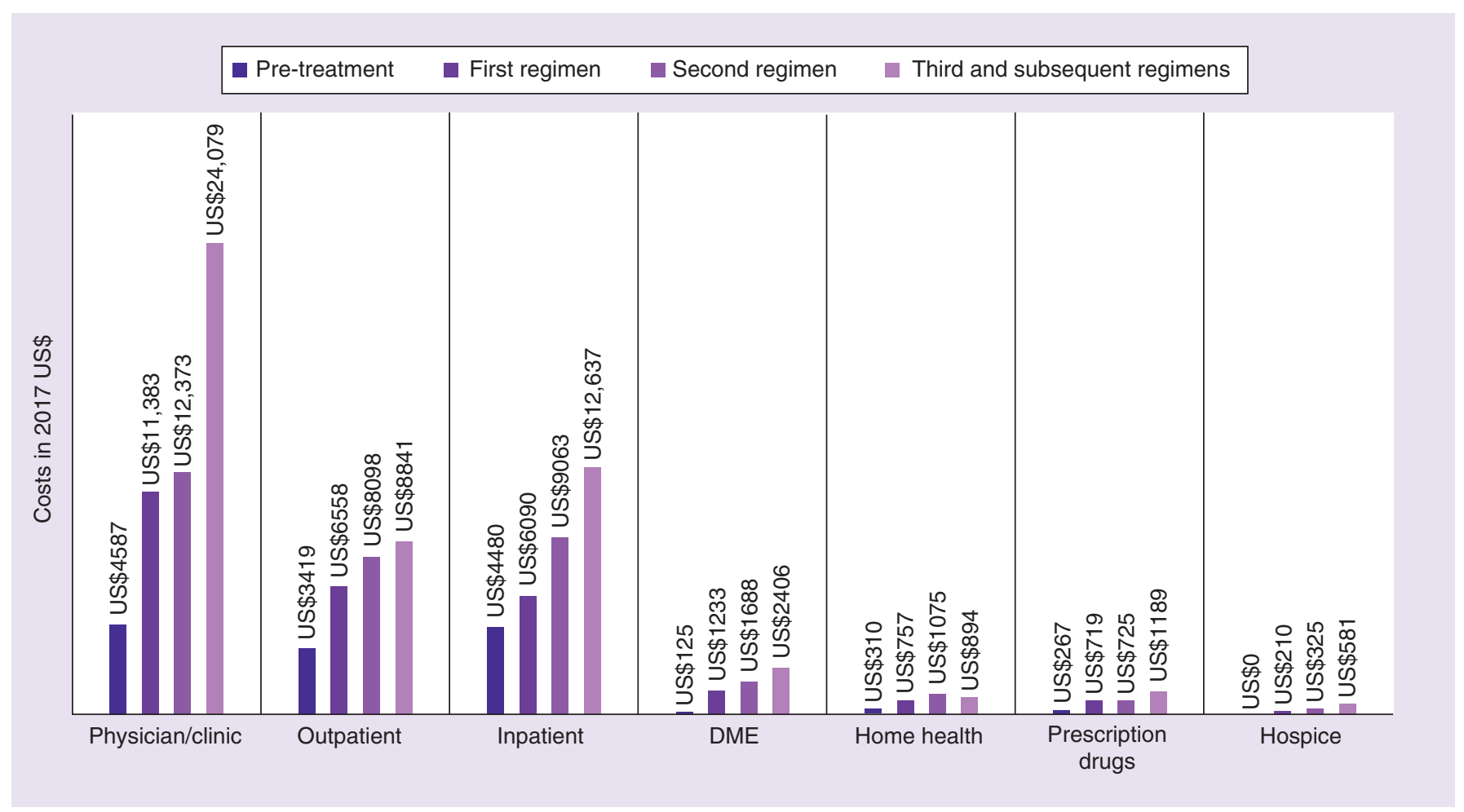

Figure 5. Mean total costs by service type incurred during each regimen sequence (main analysis). All costs presented in 2017 US dollars. DME: Durable medical equipment.

to starting treatment, US $\$ 27,000$ in the first regimen, US $\$ 33,000$ in the second regimen and US $\$ 51,000$ in the third and subsequent regimen, on a per patient basis.

Compared with a retrospective chart review analysis Bajaj et al. conducted on 411 younger patients with mTNBC, the median durations of therapy in our analysis were shorter for first (2.7 vs 7.5 months) and second regimens (3.1 vs 7.3 months) [30]. Bajaj et al. also identified more unique treatment sequences in their analysis than we did in ours (256 vs 153). This may be due to the difference in age distribution since elderly patients have more limited options than younger patients due to the toxicity profile of chemotherapy. However, the most prevalent treatment regimens in the Bajaj et al. analysis were single agent taxane (22\%) in first regimen and capecitabine (20\%) in second regimen, which was consistent with our findings. Our PPPM cost estimate (US $\$ 10,084)$ closely resembles the PPPM cost in a recent SEER-Medicare analysis by Schwartz et al. (US\$9159) [17], and is also close to the PPPM cost (US\$7271) reported by Lang et al. in stage IV estrogen receptor-positive breast cancer patients [19]. For overall survival, previous work has documented that median overall survival was between 12 and 13 months in de novo mTNBC patients receiving chemotherapy [13,31]. Berkowtiz et al. found the cost of the terminal phase (defined as the last 3 months of life) to be US $\$ 13,476$ among metastatic breast cancer patients. When the sample was restricted to patients $>60$ years than the mean terminal phase cost was found to be US\$9037 (compared with US\$14,311 in our analysis). Our numbers are higher possibly because the Berkowitz et al. estimate is dated (year 2000 vs year 2017).

Our analysis has several key strengths. First, it used a nationally representative database that represents the elderly US population with mTNBC. Given that mTNBC represents a small fraction of newly diagnosed breast cancer cases, this analysis had a higher sample size compared with previous studies. Second, the results provided herein can be used by healthcare payers to estimate the expected monthly and cumulative real-world costs that their enrollees who have mTNBC may incur at various stages of their treatment. Third, most prior research has focused on characterizing chemotherapy-treated patients. Our analysis highlights that half of mTNBC patients are not receiving chemotherapy and quantifies their economic burden; to our knowledge, this has not previously been examined. The reason why half of mTNBC patients remain chemotherapy-untreated needs further research. Such patients may be candidates for immunotherapy treatments [32,33]. 
This analysis has some limitations. Results presented here may not be generalizable to younger mTNBC patients given that the population studied was age 66 or over. Also, some treatments were not included in the treatment patterns analysis (e.g., eribulin) were not included since they were approved after 2013. In addition, the identified TNBC patients in our study may not truly be HER2 negative (HER2 status was directly available from charts only in 2010 and 2011). We assumed that those patients who were tested for HER2 and subsequently did not receive hormonal therapy were HER2 negative. While patients (especially the elderly) may not receive hormonal therapy for other reasons, our approach targets those patients who were tested for the HER2 and therefore there was intent to treat with hormonal therapy. Another limitation is that there were no quality of life data in the SEER-Medicare database. It would have been interesting to understand how more regimens and costs impact quality of life. For example, if costs incurred increase with more regimens but quality of life deteriorates, that may not be optimal from the patient's perspective. Third, the patients included in this study were diagnosed with mTNBC between 2004 and 2011. There have been changes in treatment strategies since 2011 with the advent of next-generation sequencing (NGS)-directed therapy and immunotherapy. Future studies with more updated data should conduct a comparison of survival and cost outcomes across time periods (e.g., 2004-2008 vs 2008-2012 vs 2012-2018). In the current study, a comparison of the patient cohorts diagnosed between 2004-2008 and 2008-2011 revealed similar survival and total PPPM cost estimates. Finally, there is the potential for misclassifying treatments when defining chemotherapy regimens.

\section{Conclusion}

In conclusion, about half of women with newly diagnosed mTNBC did not receive any chemotherapy and had a median overall survival of 3.5 months. The most commonly used first regimen was paclitaxel followed by capecitabine. The chemotherapy-treated group cost Medicare approximately US $\$ 13,000$ per patient from their diagnosis until the start of treatment, US $\$ 27,000$ in the first regimen, US $\$ 33,000$ in the second regimen and US $\$ 51,000$ in the third and subsequent regimens. This analysis documents an unmet medical need and calls for improving treatment rates and outcomes in elderly patients with mTNBC.

\section{Future perspective}

Future studies should assess treatment patterns among mTNBC patients following the advent of NGS-directed targeted therapy and immunotherapy. These therapies may have a differential impact on overall survival, healthcare cost, HCRU and perhaps patient quality of life.

\section{Acknowledgements}

The authors would like to acknowledge $X \mathrm{Ng}$ and $\mathrm{C}$ Zhang for providing medical writing support. This study used the linked SEER-Medicare database. The interpretation and reporting of these data are the sole responsibility of the authors. The authors acknowledge the efforts of the National Cancer Institute; the Office of Research, Development and Information, CMS; Information Management Services (IMS), Inc.; and the SEER program tumor registries in the creation of the SEER-Medicare database.

\section{Financial \& competing interests disclosure}

A Aly, R Shah and M Botteman are employees of Pharmerit International. M Botteman is also a shareholder of Pharmerit International. K Hill is an employee and has stock options in Celldex Therapeutics. The authors have no other relevant affiliations or financial involvement with any organization or entity with a financial interest in or financial conflict with the subject matter or materials discussed in the manuscript apart from those disclosed.

No writing assistance was utilized in the production of this manuscript.

Open access

This work is licensed under the Attribution-NonCommercial-NoDerivatives 4.0 Unported License. To view a copy of this license, visit http://creativecommons.org/licenses/by-nc-nd/4.0/ 
Summary points

- Triple-negative breast cancer (TNBC) is an aggressive variant of breast cancer that accounts for approximately $15 \%$ of all breast cancer cases.

- The mainstay of treatment of TNBC is conventional chemotherapy including taxanes or anthracyclines.

- While TNBC patients may respond initially to anthracycline/taxane-based adjuvant chemotherapies, they often develop visceral or CNS metastasis.

- This analysis aimed to describe the treatment patterns and overall survival of metastatic TNBC (mTNBC) patients according to the number of regimens received and examine the healthcare costs and healthcare resource use of patients with $\mathrm{mTNBC}$ according to the number of regimens received.

- SEER-Medicare database was used to identify mTNBC patients diagnosed from 2004 through 2011.

- Of 625 included mTNBC patients, the median age at diagnosis was 76 years (range: $66-102$ ) and the majority were non-Hispanic white $(72 \%)$ and had $\mathrm{CCl}>1$ (25\%).

- The proportions of patients using combination chemotherapy were higher in the second (53\%) and third (41\%) regimens compared with the first regimen (35\%).

- The median overall survival for all patients in the study sample was 7.0 (6.2-8.1) months. Median survival was 12.2 (10.7-14.9) months for all treated patients compared to 3.5 (3.2-4.3) months for untreated patients.

- The mean cumulative cost per patient was US $\$ 73,586$ for all patients and increased as patients received more regimens (no chemotherapy [NC]: US $\$ 51,070$; patients who received only one regimen [1R]: $\$ 68,899$; patients who received only two regimens [2R]: US $\$ 107,214$; patients who received three or more regimens [3R+]: US $\$ 143,150)$. However, the mean cost per patient per month (PPPM) was US $\$ 10,084$ for all patients and decreased as the number of regimens received increased (NC: US $\$ 12,101 ; 1 R$ : US $\$ 9721 ; 2 R$ : US $\$ 6853$; 3R+: US $\$ 5986$ ) Most of the PPPM costs were due to inpatient and physician costs across all groups.

- Total unadjusted costs incurred per patient during each of the phases were US $\$ 13,188$ for the pretreatment phase, US $\$ 26,950$ for the first regimen, US $\$ 33,347$ for the second regimen and US $\$ 50,627$ for third and subsequent regimens, respectively.

- Patients in the NC group had a median of one ER admission, one hospitalization (median length of stay: 6 days), and eight office visits during their follow-up period.

- This analysis documents an unmet medical need and calls for improving treatment rates and outcomes in elderly patients with mTNBC.

\section{References}

1. Cleator S, Heller W, Coombes RC. Triple-negative breast cancer: therapeutic options. Lancet Oncol. 8(3), 235-244 (2007).

2. Kohler BA, Sherman RL, Howlader N et al. Annual report to the nation on the status of cancer, 1975-2011, featuring incidence of breast cancer subtypes by race/ethnicity, poverty, and state. J. Natl Cancer Inst. 107(6), djv048 (2015).

3. SEER*Stat Database: Incidence-SEER 18 Regs Research Data + Hurricane Katrina Impacted Louisiana Cases, Nov. 2016 Sub (2000-2014) -Linked To County Attributes-Total US, 1969-2015 Counties.

4. Schwentner L, Wolters R, Koretz K et al. Triple-negative breast cancer: the impact of guideline-adherent adjuvant treatment on survival a retrospective multi-centre cohort study. Breast Cancer Res. Treat. 132(3), 1073-1080 (2012).

5. Zhang JF, Liu J, Wang Y, Zhang B. Novel therapeutic strategies for patients with triple-negative breast cancer. Oncotargets Ther. 9, 6519-6528 (2016).

6. Dent R, Trudeau M, Pritchard KI et al. Triple-negative breast cancer: clinical features and patterns of recurrence. Clin. Cancer Res. 13(15 Pt 1), 4429-4434 (2007).

7. Guarneri V, Dieci MV, Conte P. Relapsed triple-negative breast cancer: challenges and treatment strategies. Drugs 73(12), 1257-1265 (2013).

8. Kennecke H, Yerushalmi R, Woods R et al. Metastatic behavior of breast cancer subtypes. J. Clin. Oncol. 28(20), 3271-3277 (2010).

9. Andre F, Zielinski CC. Optimal strategies for the treatment of metastatic triple-negative breast cancer with currently approved agents. Ann. Oncol. 23(Suppl. 6), vi46-vi51 (2012).

10. Kassam F, Enright K, Dent $\mathrm{R}$ et al. Survival outcomes for patients with metastatic triple-negative breast cancer: implications for clinical practice and trial design. Clin. Breast Cancer 9(1), 29-33 (2009).

11. Mustacchi G, De Laurentiis M. The role of taxanes in triple-negative breast cancer: literature review. Drug Des. Devel. Ther. 9, 4303 (2015).

12. Shachar SS, Jolly TA, Jones E, Muss HB. Management of triple-negative breast cancer in older patients: how is it different? Oncology (Williston Park) 32(2), 58-63 (2018).

13. Kassam F, Enright K, Dent R et al. Survival outcomes for patients with metastatic triple-negative breast cancer: implications for clinical practice and trial design. Clin. Breast Cancer 9(1), 29-33 (2009). 
14. Liedtke C, Mazouni C, Hess KR et al. Response to neoadjuvant therapy and long-term survival in patients with triple-negative breast cancer. J. Clin. Oncol. 26(8), 1275-1281 (2008).

15. Dent R, Trudeau M, Pritchard KI et al. Triple negative breast cancer: clinical features and patterns of recurrence. Clin. Cancer Res. 13(15), 4429-4434 (2007).

16. Baser O, Wei W, Henk HJ, Teitelbaum A, Xie L. Patient survival and healthcare utilization costs after diagnosis of triple-negative breast cancer in a United States managed care cancer registry. Curr. Med. Res. Opin. 28(3), 419-428 (2012).

17. Schwartz KL, Simon MS, Bylsma LC et al. Clinical and economic burden associated with stage III to IV triple-negative breast cancer: a SEER-Medicare historical cohort study in elderly women in the United States. Cancer 124(10), 2104-2114 (2018).

18. Warren JL, Klabunde CN, Schrag D, Bach PB, Riley GF. Overview of the SEER-Medicare data: content, research applications, and generalizability to the United States elderly population. Med. Care 40(8), IV-3-IV-18 (2002).

19. Lang K, Huang H, Sasane M, Paly VF, Hao Y, Menzin J. Survival, healthcare resource use and costs among stage IV ER + breast cancer patients not receiving HER2 targeted therapy: a retrospective analysis of linked SEER-Medicare data. BMC Health Serv. Res. 14, 298 (2014).

20. Zuckerman IH, Onukwugha E, Gardner JF, McNally DL, Seal BS, Mullins CD. Characteristics of triple-negative metastatic breast cancer among older adults: a population-based analysis. J. Clin. Oncol. 28(15 Suppl.), 1092-1092 (2010).

21. Davidoff AJ, Tang M, Seal B, Edelman MJ. Chemotherapy and survival benefit in elderly patients with advanced non-small-cell lung cancer. J. Clin. Oncol. 28(13), 2191-2197 (2010).

22. Hanna N, Bikov KA, McNally D, Onwudiwe NC, Dalal M, Mullins CD. Impact of venous thromboembolism on mortality of elderly Medicare patients with stage III colon cancer. Oncologist 17(9), 1191-1197 (2012).

23. Onukwugha E, Kwok Y, Ciezki JP et al. Skeletal-related events and mortality among men diagnosed with advanced prostate cancer: the impact of alternative measures of radiation to the bone. PLoS ONE 12(4), e0175956 (2017).

24. Griffiths RI, Gleeson ML, Valderas JM, Danese MD. Impact of undetected comorbidity on treatment and outcomes of breast cancer. Int. J. Breast Cancer 2014, 970780 (2014).

25. Bikov KA, Mullins CD, Seal B, Onukwugha E, Hanna N. Algorithm for identifying chemotherapy/biological regimens for metastatic colon cancer in SEER-Medicare. Med. Care 53(8), e58-e64 (2015).

26. Liang C, Li L, Fraser CD et al. The treatment patterns, efficacy, and safety of nab ((R))-paclitaxel for the treatment of metastatic breast cancer in the United States: results from health insurance claims analysis. BMC Cancer 15, 1019 (2015).

27. Jiang S, Hill K, Varghese D, Waldeck AR, Botteman M. Treatment patterns in metastatic triple-negative breast cancer (mTNBC). J. Clin. Oncol. 35(15 Suppl.), e12589-e12589 (2017).

28. Zeichner SB, Terawaki H, Gogineni K. A review of systemic treatment in metastatic triple-negative breast cancer. Breast Cancer 10 , 25-36 (2016).

29. Tang S, Jeong JH. Median tests for censored survival data; a contingency table approach. Biometrics 68(3), 983-989 (2012).

30. Bajaj P, Latremouille-Viau D, Guerin A et al. 268P What are the treatment patterns and overall survival (OS) in patients with metastatic triple-negative breast cancer (mTNBC) in US clinical practice? Annal. Oncol. 28(Suppl. 5), mdx365.031-mdx365.031 (2017).

31. Clarke C, Chu L, Tao L et al. Abstract P3-07-26: characteristics of de novo metastatic breast cancer in California, 2005-2011. Cancer Res. 75(9 Suppl.), doi:10.1158/1538-7445.SABCS14-P3-07-26 (2015).

32. Yu LY, Tang J, Zhang CM et al. New immunotherapy strategies in breast cancer. Int. J. Environ. Res. Public Health 14(1), (2017).

33. Pusztai L, Karn T, Safonov A, Abu-Khalaf MM, Bianchini G. New strategies in breast cancer: immunotherapy. Clin. Cancer Res. 22(9), 2105-2110 (2016). 